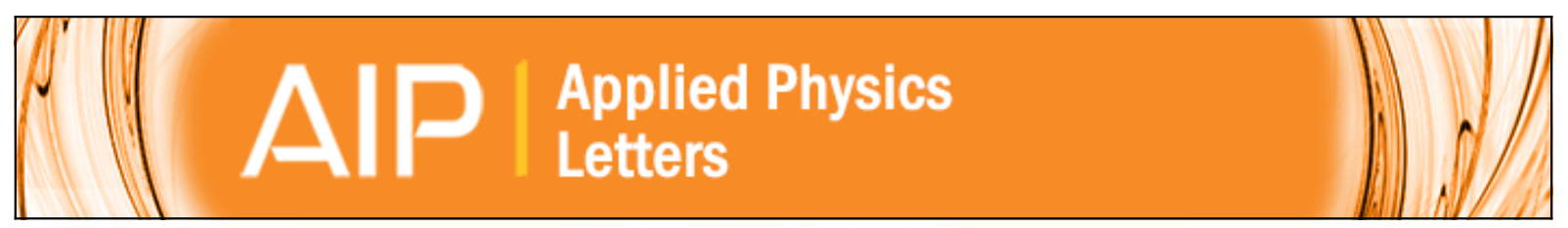

\title{
Electric field induced transformation of carbon nanotube to graphene nanoribbons using Nafion as a solid polymer electrolyte
}

M. J. Jaison, K. Vikram, Tharangattu N. Narayanan, and Vijayamohanan K. Pillai

Citation: Applied Physics Letters 104, 153111 (2014); doi: 10.1063/1.4871867

View online: http://dx.doi.org/10.1063/1.4871867

View Table of Contents: http://scitation.aip.org/content/aip/journal/apl/104/15?ver=pdfcov

Published by the AIP Publishing

\section{Articles you may be interested in}

Morphology of a graphene nanoribbon encapsulated in a carbon nanotube

AlP Advances 3, 092103 (2013); 10.1063/1.4821102

Comparison of isotope effects on thermal conductivity of graphene nanoribbons and carbon nanotubes Appl. Phys. Lett. 103, 013111 (2013); 10.1063/1.4813111

Width dependent edge distribution of graphene nanoribbons unzipped from multiwall carbon nanotubes J. Appl. Phys. 113, 174307 (2013); 10.1063/1.4803701

Interfacial thermal conductance limit and thermal rectification across vertical carbon nanotube/graphene nanoribbon-silicon interfaces

J. Appl. Phys. 113, 064311 (2013); 10.1063/1.4790367

Polymer-electrolyte gated graphene transistors for analog and digital phase detection

Appl. Phys. Lett. 99, 043307 (2011); 10.1063/1.3615247

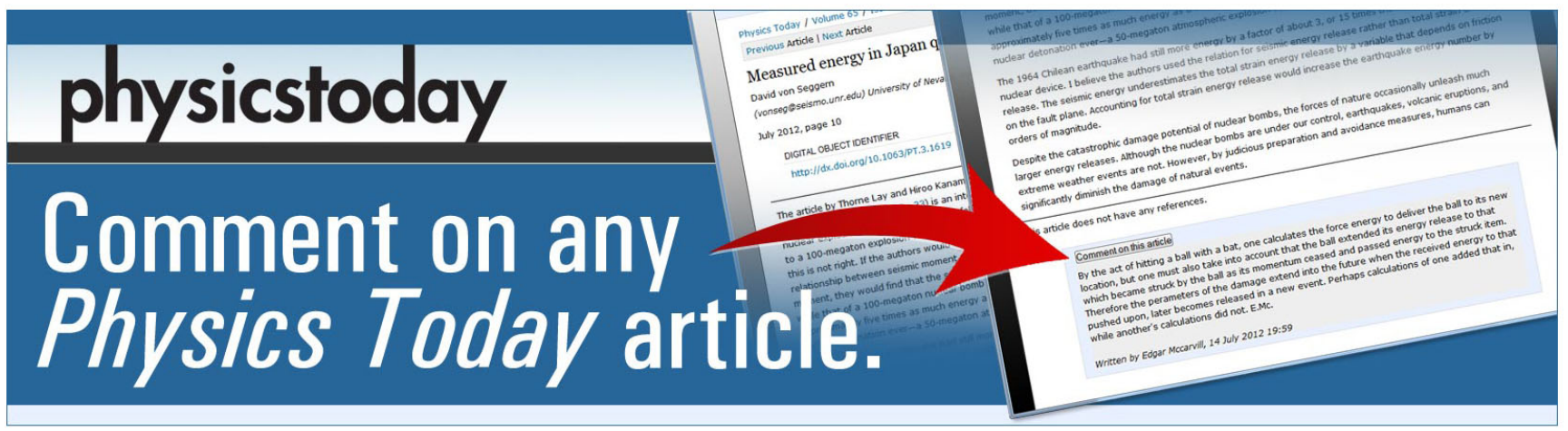




\title{
Electric field induced transformation of carbon nanotube to graphene nanoribbons using Nafion as a solid polymer electrolyte
}

\author{
M. J. Jaison, K. Vikram, Tharangattu N. Narayanan, ${ }^{a)}$ and Vijayamohanan K. Pillai ${ }^{a)}$ \\ CSIR-Central Electrochemical Research Institute (CSIR-CECRI), Karaikudi-630006, India
}

(Received 11 February 2014; accepted 7 April 2014; published online 16 April 2014)

\begin{abstract}
We report a remarkable transformation of multiwalled carbon nanotubes (MWCNTs, average diameter $40 \mathrm{~nm}$ ) to graphene nanoribbons (GNRs) in response to a field gradient of $\sim 25 \mathrm{~V} / \mathrm{cm}$, in a sandwich configuration using a solid state proton conducting polymer electrolyte like a thin perfluorosulphonated membrane, Nafion. In response to the application of a constant voltage for a sustained period of about $24 \mathrm{~h}$ at both room temperature and elevated temperatures, an interesting transformation of MWCNTs to GNRs has been observed with reasonable yield. GNRs prepared by this way are believed to be better for energy storage applications due to their enhanced surface area with more active smooth edge planes. Moreover, possible morphological changes in CNTs under electric field can impact on the performance and long term stability of devices that use CNTs in their electronic circuitry. (C) 2014 AIP Publishing LLC. [http://dx.doi.org/10.1063/1.4871867]
\end{abstract}

Graphene has emerged as one of the most promising materials to replace many active components in future devices ranging from solar cells to single electron transisters. ${ }^{1-4}$ However, its two dimensional lattice having a half-filled band with a linear energy dispersion near the first Brillouin zone effecting a zero band gap, ${ }^{5}$ hinders their use in electronic circuitries. Consequently, great efforts have been expended worldwide to open a band gap in graphene using different techniques such as creation of controlled topological defects in the lattice, substitution of hetero-elements like nitrogen, and boron, control of edge states using selected chemical species and controlling the size of graphitic domains.

One such class of graphene derivatives having tunable non-zero band gap is called graphene nanoribbons (GNRs). The unique electronics properties of GNRs are highly influenced by their aspect ratio as well as the edge states facilitating the formation of dangling bonds. To achieve large-scale production of high-quality GNRs with narrow widths, numerous fabrication strategies, including both top-down and bottom-up approaches, have been proposed. Those include lithographic patterning followed by plasma etching of graphene, ${ }^{6,7}$ sonochemical breaking of chemically derived graphene, metal-catalyzed ${ }^{8,9}$ or oxidative cutting of graphene, ${ }^{10}$ direct chemical vapour deposition (CVD), ${ }^{11}$ plasma CVD, ${ }^{12}$ chemical synthesis, ${ }^{13,14}$ and unzipping of carbon nanotubes. ${ }^{15,16}$ Recent reports by Dai et al. and Tour et al. suggest a novel concept named unzipping of carbon nanotubes (CNTs) to obtain narrow sized smooth edged GNRs. ${ }^{15,16}$ Unzipping of CNTs is one of the easy methods to generate GNRs with narrow band gap-by controlling the alignment, diameter, and length- especially from the application point of view. ${ }^{17}$ Longitudinal unzipping of CNTs with controlled alignment can be achieved with the help of laser and ion beams (FIB) cutting, plasma etching, and careful chemical oxidation. Indeed, many strong oxidizing agents have been

\footnotetext{
${ }^{\text {a) }}$ Authors to whom correspondence should be addressed. Electronic addresses: tn_narayanan@yahoo.com and vk.pillai@ncl.res.in.
}

used as a well-known strategy for the longitudinal cutting of multiwalled carbon nanotubes (MWCNTs). ${ }^{16}$ More significantly, chemical methods generate too many defect sites presumably by the over-oxidation of edges to adversely affect electronic properties of graphene such as electron mobility and conductivity. Moreover, the use of strong reducing agents might pose difficulties in controlling the layer thickness of graphene ribbons, along with other disposal concerns.

Recent reports, including that from our group, have proven electrochemical unzipping of CNTs as an efficient way to generate GNRs with controlled edge states, and demonstrated their superior behaviour over GNRs prepared by chemical methods. ${ }^{18}$ The rate of oxidation could be controlled potentiostatically during electrochemical unzipping by changing time, commensurate with the quality and yield of GNRs produced by this facile unzipping of nanotubes. ${ }^{18}$ This longitudinal unzipping of MWCNTs in aqueous media facilitating GNRs has a definite edge over other synthetic methods in term of lesser defects and edge smoothness. Interestingly, it is reported that similar unzipping in non-aqueous media forms graphene quantum dots (GQDs), where size dependant properties like luminescence and single electron transfer have been recently demonstrated. ${ }^{19,20}$ Although the use of aqueous electrolytes is known to transform MWCNTs to GNRs irrespective of the $\mathrm{pH}$, use of non-aqueous solvents such as acetonitrile and dimethylformamide generates GQDs and the reason for this difference is not yet known. More recently, ionic liquids also have been used to generate GNRs by unzipping of $\mathrm{CNT}^{21}$ Hence the mechanism of electrochemical cleavage of CNTs still remains elusive and unravelling the mechanism is important for making more controlled structures with definite edge states and defects.

Use of a solid electrolyte in an electrochemical cleavage process will have a definite merit over aqueous or nonaqueous electrolytes based unzipping due to the absence of intercalating anions and spatial confinement of the electric field, unlike in the case of liquid electrolytes. Moreover, the use of a solid electrolyte might provide valuable information 
to understand why longitudinal unzipping occurs in certain class of electrolytes to form GNRs whereas similar electric field in non-aqueous electrolytes almost invariably generates GQDs. Further, it is important to realise the role of electric field in inducing the transformational changes in CNTs for their extensive use in electronic circuitries as interconnecting conductive channels or active semiconducting elements.

In this Letter, we demonstrate the unzipping of MWCNTs to GNRs in a sandwiched structure containing copper $(\mathrm{Cu})$ electrodes separated by a solid polymer electrolyte (Nafion membrane) modified with MWCNTs on its either sides. On applying a gradient electric field, this study reveals the transformation of MWCNTs (in both + ve and -ve electrodes) to longitudinally unzipped GNRs. This report on the unzipping of CNTs using solid state electrolytes to generate GNRs also yields considerably good yield by modulating the electric field. Use of a thin compact polymer electrolyte membrane, instead of a corrosive and voluminous liquid electrolyte, can bring several advantages in terms of easy processing and flexibility assuming that quality of GNRs is not adversely affected.

Nafion membrane consists of polytetrafluoroethylene (PTFE) backbone with side chains ending in sulphonate group which helps in sustaining proton transport across the membrane. ${ }^{22}$ Water uptake of these membranes which provides proton conductivity makes it accessible for the fuel cell and electrolyser applications. ${ }^{23}$ Nafion 117 membrane (from DuPont) was used with following standard treatment. ${ }^{24}$ Membrane was boiled for half an hour in $3 \% \mathrm{H}_{2} \mathrm{O}_{2}$ solution and then washed with deionised water. This membrane was kept in $0.5 \mathrm{M} \mathrm{H}_{2} \mathrm{SO}_{4}$ at $80^{\circ} \mathrm{C}$ for overnight to acidify the membrane. After this treatment, the membrane was kept in deionised water until further use.

MWCNT, (purchased from Nanocyl), having an average diameter of around $40 \mathrm{~nm}$ (size ranges from $10-60 \mathrm{~nm}$ ) was used to make a CNT ink with $5 \mathrm{wt}$ \% Nafion solution in isopropanol. A compact coating of MWCNT on Nafion membrane was achieved by brush coating. The reported Nafion conductivity (fully hydrated) is around $0.10 \mathrm{Scm}^{-1} .^{25}$ MWCNT coated Nafion membrane was sandwiched between two Copper plates $(1.5 \mathrm{~mm}$ thickness) in such a way that only coated portions are made in contact with $\mathrm{Cu}$ plates (Figure 1). A potential of $5 \mathrm{~V}$ was applied to the $\mathrm{Cu}$ plates for about $24 \mathrm{~h}$ at $80^{\circ} \mathrm{C}$ and MWCNT from the both sides of Nafion membrane scratched out separately. The samples collected from the both sides of the membrane are washed with isopropanol, ethanol and centrifuged. Then these samples were dried in a vacuum oven and further characterised. The percentage of MWCNTs unzipped is around $2 \%$ by weight. As in earlier reports, most of the electrochemical unzipping of CNTs were carried out at around $1-2 \mathrm{~V} \cdot{ }^{18,19}$ Because of high resistance showed by the Nafion membrane along the thickness, to alleviate IR drop problem, we have applied a potential of $5 \mathrm{~V}$ for $24 \mathrm{~h}$. We have noticed a significant reduction in current density from onset to the end of electrochemical process. This could be due to the introduction of oxygen containing groups on surface and edges of unzipped MWCNT that quench the electrical conductivity. Mechanism of MWCNT unzipping to GNRs is still on debate. Most of the reports show unzipping starts from the topological defects found in MWCNT rather

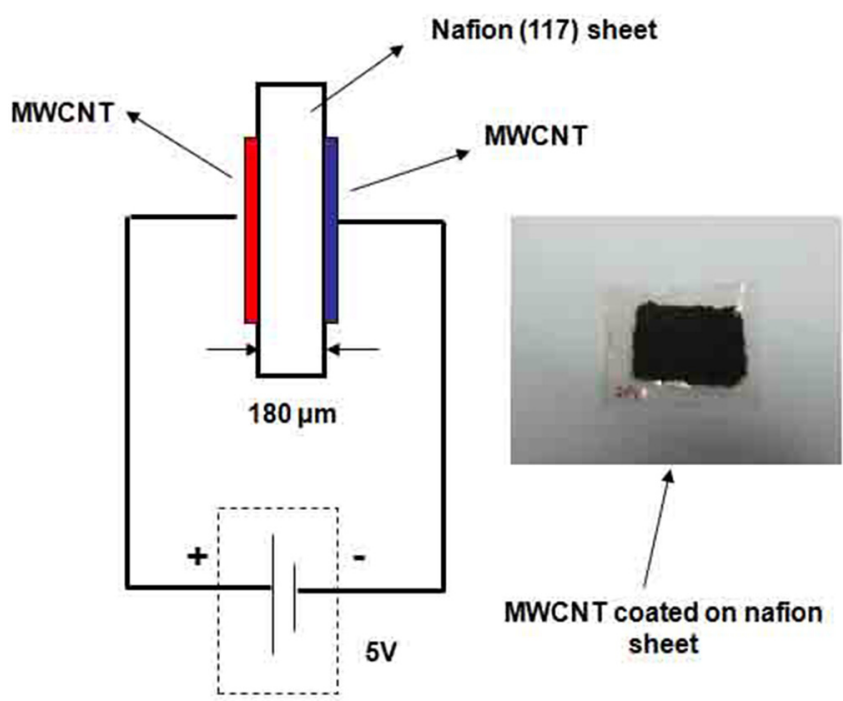

FIG. 1. Schematic diagram for experimental set up for electrochemical experiment and image of Nafion membrane coated with MWCNT.

than from edges. ${ }^{26}$ However, high electric field applied to the MWCNTs, irrespective of the polarity of electrodes, also facilitates the longitudinal or lateral unzipping. A plausible mechanism for field assisted unzipping is narrated in Figure 2. The red sites shown in Figure 2 indicate the topological defects in CNTs (details of a plausible mechanism are discussed during the end of the manuscript), where the unzipping starts, and the electric field will guide them to unzip along the axis. This is also supported by a recent theoretical model where generation of defects will impart stress on $\mathrm{sp}^{2}$ carbon matrix, ${ }^{27}$ and this will steer unzipping along the axis.

Figure 3 shows TEM images of bare MWCNT and GNRs (taken after $24 \mathrm{~h}$ electrochemical treatment). The TEM images of GNRs (Figures 3(b) and 3(c)) show that the CNT layers were unwrapped/cut/unzipped along the tube axis. This longitudinal cutting leads to the formation of high aspect ratio ribbons ( $\sim 150 \mathrm{~nm}$ length and $\sim 33 \mathrm{~nm}$ width) with thickness few nanometres $(\sim 5-7 \mathrm{~nm})$. Figure 3(b)-inset shows the width distribution of GNRs, indicating the width ranging from $20 \mathrm{~nm}-60 \mathrm{~nm}$ centred at $33 \mathrm{~nm}$. Figure 3(c)inset depicts the mechanism of longitudinal cutting which is agreement with the TEM image shown in Figure 3. An HRTEM image taken from the edge of a GNR indicates that the inter layer spacing is $\sim 0.59 \mathrm{~nm}$, and it also shows the presence of many layers in the GNRs.

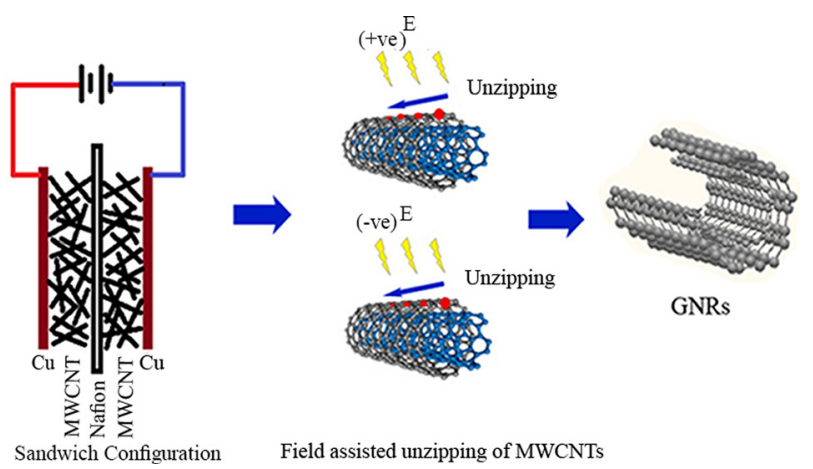

FIG. 2. Schematic representation of electric field assisted unzipping of MWCNTs in a solid polymer electrolyte. 

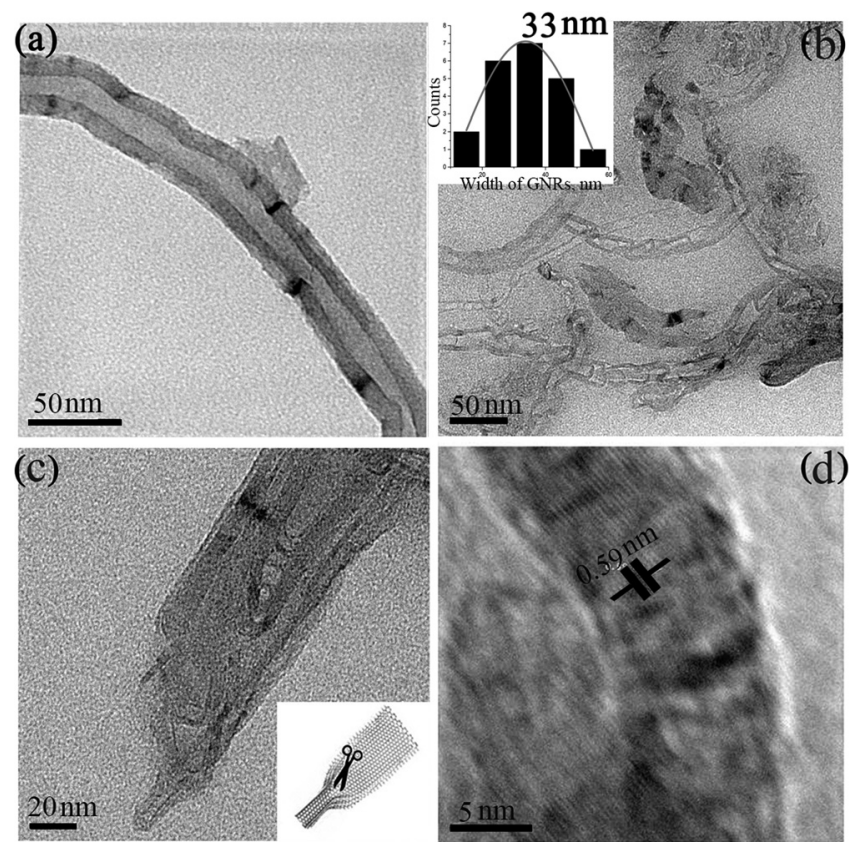

FIG. 3. Typical TEM images of (a) pristine MWCNT, (b) and (c) unzipped MWCNT showing the longitudinal cutting during the electrochemical process in solid polymer electrolyte, ((b)-inset) width distribution histogram of GNRs, and ((c)-inset) schematic of longitudinal unzipping of MWCNTs.(d) TEM image of unzipped MWCNT showing fringes of graphene sheets having a calculated inter-planar spacing of $0.59 \mathrm{~nm}$.

FT-IR is used to investigate the functional groups attached to the MWCNTs before and after electrochemical treatment (Figure 4). It is possible that more number of hydroxyl groups could be generated during the temperature assisted electrochemical unzipping of MWCNTs. ${ }^{28}$ Here, a significant enhancement in the intensity of hydroxyl $(-\mathrm{OH})$ stretching is observed in the case of GNRs, a broad peak at $3447 \mathrm{~cm}^{-1}$, on comparison with pristine MWCNTs. FT-IR band at 2861 and $2904 \mathrm{~cm}^{-1}$ are corresponding to the $-\mathrm{CH}_{2}$ symmetric and asymmetric stretching, respectively, indicating the presence of hydrogen on edge carbon atoms. In addition, a peak arises at $1635 \mathrm{~cm}^{-1}$ attributed to carbonyl $(-\mathrm{C}=\mathrm{O})$ stretching, which is present in both bare MWCNTs and GNRs, indicating that the formation of some oxygen functionalities like acidic and aldehyde groups. This additional peak in bare MWCNTs may arouse due to the acidic groups introduced while they are treated with acid for catalyst purification. In comparison with bare MWCNT, there is a significant enhancement in intensities of peaks at $1720 \mathrm{~cm}^{-1}, \mathrm{COO}-$ stretching and at $3447 \mathrm{~cm}^{-1}$, -OH stretching peak, indicating the increase in the number of carboxyl groups $(-\mathrm{COOH})$ on GNRs by electric field treatment. Moreover, a prominent peak at $1049 \mathrm{~cm}^{-1}$ corresponds to $-\mathrm{C}-\mathrm{O}$ stretching in MWCNTs is quenched in GNRs. The emergence of new peaks in GNRs, and difference in the spectra of GNRs and MWCNTs reveal that a process of oxidative cutting of MWCNT is leading to the formation of additional hydroxyl, carbonyl, and carboxyl groups in GNRs.

Raman spectroscopy is widely used for the characterisation of carbonaceous materials like graphene, CNT, etc. ${ }^{29}$ Comparing the Raman spectra of both bare MWCNT and GNRs, a significant increase in the intensity of disorder (D) band is evident along with a concomitant decrease in the
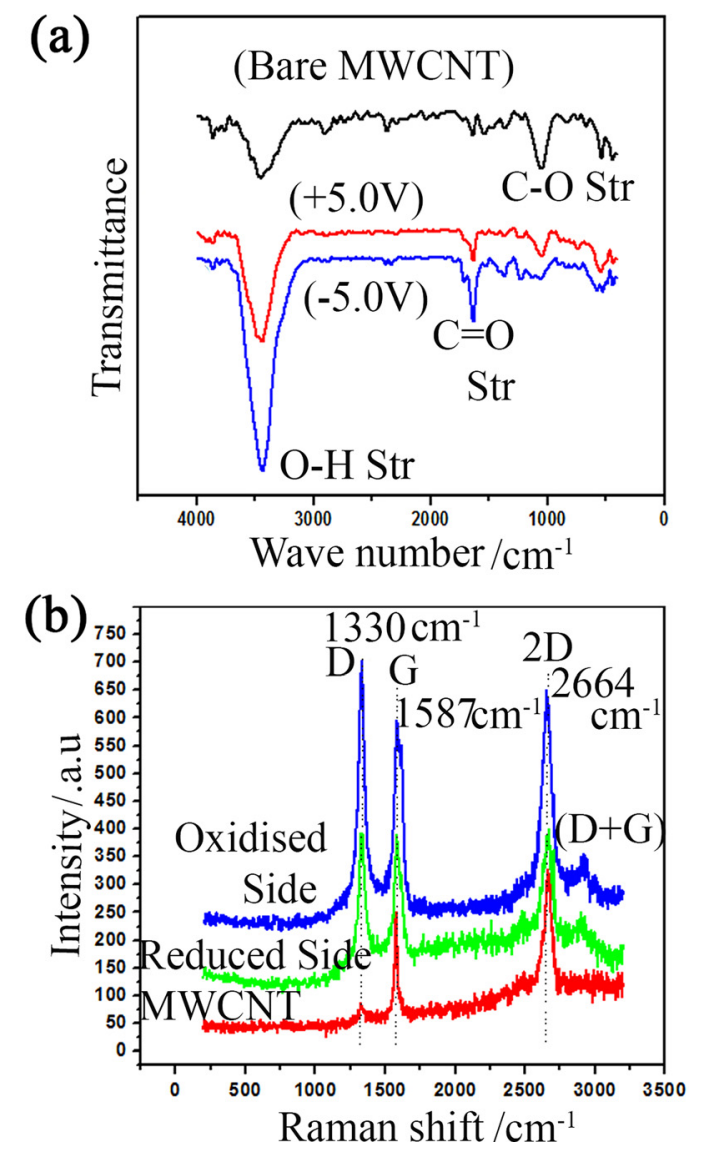

FIG. 4. (a) Comparative study of FT-IR spectra of electrochemically treated MWCNT from the both side of Nafion membrane with bare MWCNT. (b) Raman spectra of electrochemically treated MWCNT from the both side of sheet with pristine MWCNT. MWCNT's D, G, 2D, and D+G peaks are shown in order (from left) in the spectra.

intensity of second harmonic $(2 \mathrm{D}) /$ order $(\mathrm{G})\left(\mathrm{I}_{2 \mathrm{D}} / \mathrm{I}_{\mathrm{G}}\right.$ ratio) after unzipping. The $\mathrm{I}_{2 \mathrm{D}} / \mathrm{I}_{\mathrm{G}}$ values are $1.13,1.08$, and 0.86 , respectively, for pristine MWCNTs, Oxidised and Reduced MWCNTs. Additionally, an extra defect-induced combination peak $(D+G)$ around $2930 \mathrm{~cm}^{-1}$ is appeared for GNRs and this in tune with that in the earlier reports. ${ }^{11}$ These effects are attributed to the increase in the fraction of edge carbon along with the enhancement in the D band intensity with the introduction of extensive damage or defects over the edges. Moreover, introduction of carbonyl groups on edges and/or surfaces of GNRs can also enhance the intensity of D band. Quantification of the degree of disorder in the graphitic carbon material is the ratio of intensities of $D$ band to the $G$ band. The intensity ratio $\left(\mathrm{I}_{\mathrm{D}} / \mathrm{I}_{\mathrm{G}}\right)$ for GNRs increases on comparing with that of bare MWCNT. The Raman spectra of pristine MWCNT has $\mathrm{I}_{\mathrm{D}} / \mathrm{I}_{\mathrm{G}}$ value of $\sim 0.18$ while those of GNRs from the $+5 \mathrm{~V}$ and $-5 \mathrm{~V}$ sides are 1.31 and 1.03 , respectively. This increase in $\mathrm{I}_{\mathrm{D}} / \mathrm{I}_{\mathrm{G}}$ ratio indicates the possible oxidative unzipping of CNTs as suggested by other theoretical reports, ${ }^{30}$ and these results on Raman data of CNTs and GNRs are in tune with the studies conducted by Dai et al. ${ }^{31}$

To further probe the introduction of defects, surface functionalization and change in morphology of MWCNTs after electrochemical treatment, cyclic voltammetric studies were conducted on both pristine MWCNTs and GNRs. Figure 5 represents the cyclic voltammograms of MWCNTs and GNRs $v s$ mercury/mercurous sulfate (MMS) in $0.5 \mathrm{M} \mathrm{H}_{2} \mathrm{SO}_{4}$ 


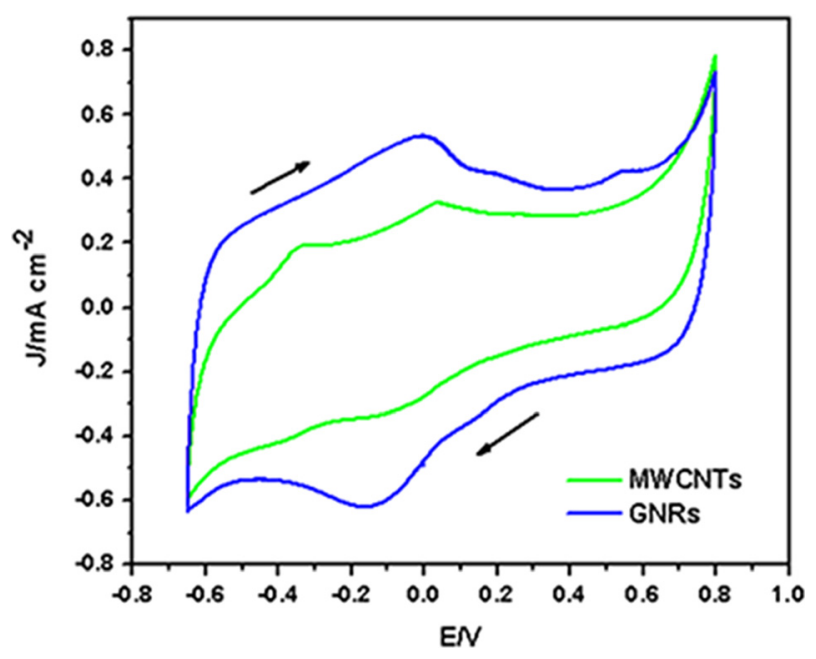

FIG. 5. Typical cyclic voltammograms of MWCNTs and GNRs in the potential window from -0.65 to $0.80 \mathrm{~V}$ vs. MMS in $0.5 \mathrm{M} \mathrm{H}_{2} \mathrm{SO}_{4}$ using glassy carbon electrode at a scan rate of $100 \mathrm{mV} / \mathrm{s}$.

using glassy carbon electrode at $100 \mathrm{mV} / \mathrm{s}$ scan rate. The ink solution made from MWCNTs and isopropanol (2 mg of MWCNTs in $1 \mathrm{ml}$ isopropanol) was drop casted on a glassy carbon electrode and this modified electrode are used for further electrochemical studies. A modified electrode with GNRs was prepared in the similar fashion. The cyclic voltammograms of both the electrodes $\left(2^{\text {nd }}\right.$ cycle) are shown in Figure 5. Interestingly, GNRs modified electrode have a high non-faradic contribution (Figure 5, blue) compared to that of the MWCNTs modified one. Morphological changes occurred during the reaction like opening of CNTs and increased exposed surface area are reflected in the cyclic voltammogram of GNRs, denoted with this increase in non-faradaic contribution. A significant enhancement in redox peaks appeared at $0.05 \mathrm{~V} v s \mathrm{MMS}$, already reported, ${ }^{32}$ indicating the contribution from Faradaic currents. Hence, change in the morphology of MWCNTs by applying electric field (TEM images, and voltammogram (increase in surface area) studies and introduction of oxygen functionalities (FT-IR, Raman, and voltammogram studies) in GNRs upon electric field are evident from these analysis. Hence these results indicate the possibilities of oxidative unzipping of MWCNTs along the longitudinal axis resulting to the formation of unzipped CNTs or GNRs.

CNT unzipping and exfoliation can be explained by several mechanisms. ${ }^{16,33}$ In most of cases, a threshold energy (around $\sim 0.60 \mathrm{eV}$ ) is generated on the surface or tip of the nanotubes to initiate the opening/cutting the CNTs. ${ }^{34}$ Recent studies based on Density Functional Theories show that a controlled external electric field on CNTs can generate this energy and can form GNRs with smoother edges. ${ }^{30}$ Furthermore, attack of oxygen adatoms on MWCNTs will assist the conversion of internal $\mathrm{C}-\mathrm{C}$ bonds to $\mathrm{C}=\mathrm{O}$ via epoxy linkage. In the present case, both electric field and presence of oxygen on pristine MWCNTs (-OH stretching at $3447 \mathrm{~cm}^{-1}$ ), accompanied by a stress along the longitudinal direction forced to unzip in an ordered sequential manner to GNRs. It is also observed that temperature has insignificant role in this unzipping process. We have carried out the unzipping experiment with $\left(80^{\circ} \mathrm{C}\right)$ and without (room temperature unzipping) the application of temperature. We observed significant unzipping in both the cases giving similar types of GNRs shown in Figure 3. In electrochemical process, an applied voltage in presence of suitable electrolyte generates enough electric field on electrode/electrolyte interface is assumed to be the cause of unzipping in CNT. In our earlier studies, we have achieved the longitudinal unzipping by electrochemical method in aqueous solution. ${ }^{18}$ In the electrochemical process with Nafion membrane, high electric field on CNT/Nafion interface acquires energy to break the $\mathrm{C}-\mathrm{C}$ bonds to achieve unzipping in CNT. We speculate that cleavage of $\mathrm{C}-\mathrm{C}$ bond generates short spanned intermediates (anionic and cationic radicals). Recently, L. Jiang et al. ${ }^{35}$ shown the formation of graphene via radical coupling reaction, support our mechanism of unzipping. In situ studies on the formation of these reactive radicals will further probe the mechanism and these studies are going on.

In conclusion, GNRs are formed from MWCNTs via an electrochemical process, in which a solid polymer electrolyte has been used. The oxidative unzipping of MWCNTs is achieved by the electric field gradient generated on the Nafion/MWCNT interface. TEM results indicate the longitudinal cutting of MWCNTs giving long and high aspect ratio GNRs. FT-IR, and cyclic voltammetry data indicate the presence of larger number of oxygen species on GNR's surface than initial MWCNTs. These results indicate that electric field can make structural changes in CNTs resulting to the formation of other structures having different morphology, and electrochemical activity.

The authors thank CIF,CSIR-CECRI staff Mr. A. Rathish Kumar for TEM analysis. M. J. Jaison acknowledges University Grants Commission for providing JRF fellowship and also acknowledges the tremendous support received from Dr. T. Premkumar, CSIR-CECRI, during this work. V. K. Pillai thanks the financial support from CSIR through MULTIFUN (CSC0101).

${ }^{1}$ H. Santos, L. Chico, and L. Brey, Phys. Rev. Lett. 103, 086801 (2009).

${ }^{2}$ F. Schwierz, Nat. Nanotechnol. 5, 487 (2010).

${ }^{3}$ J. G. Son, M. Son, K.-J. Moon, B. H. Lee, J.-M. Myoung, M. S. Strano, M.-H. Ham, and C. a Ross, Adv. Mater. 25, 4723 (2013).

${ }^{4}$ Z. Yang, M. Liu, C. Zhang, W. W. Tjiu, T. Liu, and H. Peng, Angew. Chem., Int. Ed. Engl. 52, 3996 (2013).

${ }^{5}$ A. K. Geim and K. S. Novoselov, Nature Mater. 6, 183 (2007).

${ }^{6}$ M. Han, B. Özyilmaz, Y. Zhang, and P. Kim, Phys. Rev. Lett. 98, 206805 (2007).

${ }^{7}$ Z. Chen, Y.-M. Lin, M. J. Rooks, and P. Avouris, Phys. E 40, 228 (2007).

${ }^{8}$ S. S. Datta, D. R. Strachan, S. M. Khamis, and A. T. C. Johnson, Nano. Lett. 8, 1912 (2008).

${ }^{9}$ L. Ci, Z. Xu, L. Wang, W. Gao, F. Ding, K. F. Kelly, B. I. Yakobson, and P. M. Ajayan, Nano Res. 1, 116 (2008).

${ }^{10}$ S. Fujii and T. Enoki, J. Am. Chem. Soc. 132, 10034 (2010).

${ }^{11}$ J. Campos-Delgado, J. M. Romo-Herrera, X. Jia, D. a Cullen, H. Muramatsu, Y. A. Kim, T. Hayashi, Z. Ren, D. J. Smith, Y. Okuno, T. Ohba, H. Kanoh, K. Kaneko, M. Endo, H. Terrones, M. S. Dresselhaus, and M. Terrones, Nano Lett. 8, 2773 (2008).

${ }^{12}$ T. Kato and R. Hatakeyama, Nat. Nanotechnol. 7, 651 (2012).

${ }^{13}$ X. Yang, X. Dou, A. Rouhanipour, L. Zhi, H. J. Räder, and K. Müllen, J. Am. Chem. Soc. 130, 4216 (2008).

${ }^{14}$ J. Cai, P. Ruffieux, R. Jaafar, M. Bieri, T. Braun, S. Blankenburg, M. Muoth, A. P. Seitsonen, M. Saleh, X. Feng, K. Müllen, and R. Fasel, Nature 466, 470 (2010).

${ }^{15}$ L. Jiao, L. Zhang, X. Wang, G. Diankov, and H. Dai, Nature 458, 877 (2009). 
${ }^{16}$ D. V. Kosynkin, A. L. Higginbotham, A. Sinitskii, J. R. Lomeda, A. Dimiev, B. K. Price, and J. M. Tour, Nature 458, 872 (2009).

${ }^{17}$ L. Ma, J. Wang, and F. Ding, Chem. Phys. Chem. 14, 47 (2013).

${ }^{18}$ D. B. Shinde, J. Debgupta, A. Kushwaha, M. Aslam, and V. K. Pillai, J. Am. Chem. Soc. 133, 4168 (2011).

${ }^{19}$ D. B. Shinde and V. K. Pillai, Chemistry 18, 12522 (2012).

${ }^{20}$ D. B. Shinde and V. K. Pillai, Angew. Chem., Int. Ed. Engl. 52, 2482 (2013).

${ }^{21}$ S. Vadahanambi, J.-H. Jung, R. Kumar, H.-J. Kim, and I.-K. Oh, Carbon 53, 391 (2013).

${ }^{22}$ A. Eisenberg and H. L. Yeager, "Perfluorinated Ionomer Membranes," ACS Symposium Series 180 (American Chemical Society, Washington, DC, 1982).

${ }^{23}$ M. Rikukawa and K. Sanui, Prog. Polym. Sci. 25, 1463 (2000).

${ }^{24}$ E. A. Ticianelli, J. Electrochem. Soc. 135, 2209 (1988).

${ }^{25}$ Q. Li, R. He, J. O. Jensen, and N. J. Bjerrum, Chem. Mater. 15, 4896 (2003).
${ }^{26}$ Y.-R. Kang, Y.-L. Li, and M.-Y. Deng, J. Mater. Chem. 22, 16283 (2012).

${ }^{27}$ S. Bhowmick and U. V. Waghmare, Phys. Rev. B 81, 155416 (2010).

${ }^{28}$ S. Hussain, J. Mod. Phys. 02, 538 (2011).

${ }^{29}$ A. Jorio, E. H. M. Ferreira, L. G. Cançado, C. A. Achete, and R. B. Capaz, Physics and Applications of Graphene-Experiments, edited by Dr. S. Mikhailov, (InTech, 2011), p. 439.

${ }^{30}$ C. Chen, L. Miao, K. Xu, J. Yao, C. Li, and J. Jiang, Phys. Chem. Chem. Phys. 15, 6431 (2013).

${ }^{31}$ L. Jiao, L. Zhang, L. Ding, J. Liu, and H. Dai, Nano Res. 3, 387 (2010).

${ }^{32}$ Y. Shao, J. Wang, M. Engelhard, C. Wang, and Y. Lin, J. Mater. Chem. 20, 743 (2010).

${ }^{33}$ J.-L. Li, K. Kudin, M. McAllister, R. Prud'homme, I. Aksay, and R. Car, Phys. Rev. Lett. 96, 176101 (2006).

${ }^{34}$ F. Li, E. Kan, R. Lu, C. Xiao, K. Deng, and H. Su, Nanoscale 4, 1254 (2012).

${ }^{35}$ L. Jiang, T. Niu, X. Lu, H. Dong, W. Chen, Y. Liu, W. Hu, and D. Zhu, J. Am. Chem. Soc. 135, 9050 (2013). 